\title{
Molecular identification of some wild medicinal macrofungi from Northern Iran
}

\section{Alimadadi $\mathbf{N}^{1}$, Pourianfar $\mathrm{HR}^{2}$, Amoozegar MA ${ }^{1,3^{*}}$, Zabihi SS ${ }^{1}$, Mahdizadeh $\mathbf{V}^{1}$ and Shahzadeh Fazeli SA ${ }^{1}$}

\author{
${ }^{1}$ Microorganisms Bank, Iranian Biological Resource Center (IBRC), Academic Center for Education, Culture and \\ Research (ACECR)-Tehran, Iran \\ ${ }^{2}$ Industrial Fungi Biotechnology Research Department, Research Institute for Industrial Biotechnology, Academic \\ Center for Education, Culture and Research (ACECR)- Khorasan Razavi, P.O.Box: 91775-1376, Mashhad, Iran \\ ${ }^{3}$ Extremophiles Laboratory, Department of Microbiology, Faculty of Biology and Center of Excellence in Phylogeny of \\ Living Organisms, College of Science, University of Tehran, Iran
}

Alimadadi N, Pourianfar HR, Amoozegar MA, Zabihi SS, Mahdizadeh V, Shahzadeh Fazeli SA 2019 - Molecular identification of some wild medicinal macrofungi from Northern Iran. Studies in Fungi 4(1), 26-36, Doi 10.5943/sif/4/1/4

\begin{abstract}
In last decades, macrofungi have attracted increasing attention because of their valuable nutritional and medicinal properties. In this study, a total of 180 macrofungal samples were collected from forests in Mazandaran province, Iran. The dominant orders were Polyporales (51\%) and Agaricales (35\%). Pure mycelial cultures were successfully obtained from 91 collected samples. Regarding morphological data, 47 isolates were selected for molecular identification based on internal transcribed spacer region (ITS) sequence analysis. The results showed that the 38 macrofungal isolates were belonging to 22 species, 19 genera, 10 families and 5 orders. Most of the macrofungi (47\%) were identified as Trametes species and Ganoderma species. Three isolates identified as Hohenbuehelia species, Polyporellus brumalis and Ceriporia lacerata were records as a new to the Iran fungal flora. This study increases the knowledge on Iranian macrofungal diversity and facilitates future genetic and biotechnological investigations on these macrofungi.
\end{abstract}

Key words - Internal transcribed spacer region - Iran - Macrofungi - Mazandaran province

\section{Introduction}

Macrofungi are defined as fungi that produce fruiting bodies visible without the aid of a microscope (Redhead 1997). They are extremely diverse in different climate and geographical regions in the world. It is estimated that there are 150,000-160,000 marofungal species present on the earth, among them 16,000 species have been described till date (Hawksworth 2012, Wasser 2010). The macrofungi play a vital role as decomposers, parasites, and symbionts and diet sources in ecosystems (Redhead 1997). In addition, several macrofungi have been used as a source of food and medicine for thousands of years in different civilizations (Money 2016). In last decades, the beneficial property of macrofungi has gained increasing attention by scientific community. Fruiting bodies and mycelia of macrofungi are rich in compounds with nutritional and/or medicinal properties (Cheung 2010, Reis et al. 2017). They are excellent sources of fiber, protein, essential oils, vitamins, minerals and biologically active metabolites such as phenolic compounds, terpenoids, 
polysaccharides, lectins, steroids, lipids, peptides and glycoproteins (El Enshasy \& Hatti-Kaul 2013, Rathore et al. 2017, Roncero-Ramos \& Delgado-Andrade 2017). Consequently, more than 100 medicinal functions have been attributed to macrofungi including antimicrobial, antiviral, anticancer, antioxidant, immune-modulatory, immune-suppressive, anti-allergic, hepato-protective, antidiabetic, anti-cholesterol and detoxification activities (Roncero-Ramos \& Delgado-Andrade 2017, Roupas et al. 2012, Wasser 2014). Several macrofungal compounds have been subjected to clinical trials and are used extensively in Asia to treat various diseases (Wasser 2014). Moreover, macrofungi have applications as biocontrol agents and in cosmetics (Sivanandhan et al. 2017, Taofiq et al. 2016).

Accurate identification of macrofungi is important for evaluate their diversity and applications. However, there are many reports on biodiversity and biological activity of wild macrofungi without proper authentication (Wasser 2014). Morphological characterization is common method for identification of macrofungi but it is difficult, time consuming and may lack precision in differentiating closely related species. Molecular techniques are fast and reliable and extensively are used for identification of microorganisms (Bisen et al. 2012). Among DNA barcodes, the nuclear ribosomal internal transcribed spacer (ITS) region has proved to have the highest probability of successful identification for the broadest range of fungi including macrofungi (Dentinger et al. 2011, Schoch et al. 2012).

Mazandaran province in the north of Iran is located between Albourz Mountains and Caspian Sea. Heavy to moderate rainfall, mild temperature, and dense vegetation in the forests of this region provide suitable conditions for growth of diverse fungal flora. A number of reports have been published on diversity of macrofungi in this region but most of them are based on morphological characterization (Asef \& Etemad 2016, Borhani et al. 2010, Keypour et al. 2014) and still little data is available in the literature (Rezaeian et al. 2016). However, considering the importance of native populations of macrofungi in biomedical research, breeding programs and biodiversity studies, it is essentially required to preserve the samples alive under standard conditions to maintain their original properties. Therefore, this study was conducted with the aim of collection, mycelial culture and molecular identification of wild macrofungi, with emphasis on medicinal ones, from Mazandaran province.

\section{Materials \& Methods}

\section{Collection of macrofungi}

Macrofungal specimens were collected from forests in Mazandaran province during the rainy seasons (spring and autumn) between 2015 and 2016. Detailed site information regarding location and elevation are provided in Table 1. The morphological and ecological characteristics of the macrofungi were recorded and photographed in the field. All specimens were labeled, placed in paper bags, and transferred to the laboratory for further study. Initial identification of the macrofungi carried out based on the macroscopic and microscopic characteristics using key provided by Moser (1983), Ryvarden (1991) and Ryvarden \& Melo (2014).

\section{Mycelial culture}

Using aseptic tissue culture technique, small cut pieces of fruiting bodies of the macrofungi were inoculated on malt extract agar ( $2 \%$ malt extract, $2 \%$ agar) and compost extract agar media supplemented with $0.02 \%$ chloramphenicol and incubated under dark condition for two weeks at $25^{\circ} \mathrm{C}$. Compost extract agar was prepared as detailed by Masoumi et al. (2015). Pure cultures were obtained by inoculation of $1 \mathrm{~cm}^{2}$ disks from the leading edges of the mycelia on potato dextrose agar and incubation at the same condition mentioned above. The culture of all identified macrofungi were deposited at Microorganisms Bank of Iranian Biological Resource Center (IBRC-M), Iran.

\section{DNA extraction}

For DNA extraction, the pure mycelial cultures were grown in a liquid medium $(0.5 \%$ yeast extract, $1 \%$ peptone and $2 \%$ glucose) for $4-7$ days at $25{ }^{\circ} \mathrm{C}$. DNA extracted from a small clump of 
the mycelia using the salting-out protocol described by Saba et al. (2016). The quality of DNA was analyzed by agarose gel (1\%) electrophoresis stained with ethidium bromide. DNA samples were stored at $-20{ }^{\circ} \mathrm{C}$ until use.

\section{ITS region sequencing and phylogenetic analysis}

The sequences of the internal transcribed spacer (ITS) regions (including 5.8S rDNA) determined from polymerase chain reaction (PCR) products amplified from the DNA samples using the primers ITS1 and ITS4 (White et al. 1990). DNA sequencing was carried out using Sanger (dideoxy) method with the mentioned primers. The sequences were assembled using ChromasPro software version 1.5. The sequences were compared pairwise using Basic Local Alignment Search Tool (BLAST) and aligned all sequences (retrieved from GenBank and CBS databases) using the CLUSTAL W (Thompson et al. 1994). Phylogenetic trees were reconstructed using the neighbourjoining algorithm of MEGA 7.0.21 (Kumar et al. 2016). Confidence levels of the clades were estimated from bootstrap analysis based on 1000 replications (Felsenstein 1985).

\section{Results}

A total number of 180 macrofungal specimens were collected from forests in Mazandaran province. Based on initial morphological characterization, the macrofungi belonged under 2 phyla, 6 orders, 21 families and 38 genera (Table 1). Most of the macrofungi (97\%, 175 samples) were classified under the phylum Basidiomycota, whereas others (3\%, 6 samples) belonged to the phylum Ascomycota. The dominant orders were Polyporales (51\%) and Agaricales (35\%). Other macrofungi were distributed in the orders viz. Russulales, Hymenochaetales, Auriculariales, Xylariales and Pezizales. The genera Trametes (27\%) and Ganoderma (21\%) were frequent macrofungi among the collected samples.

Pure mycelial cultures were successfully obtained from 91 collected macrofungi, while other specimens did not form mycelia or their cultures were contaminated by other fungi or bacteria. Fortyseven isolates were selected for molecular identification (Table 1) and their ITS region (including 5.8S rRNA gene) were amplified and sequenced. The PCR amplification using primers ITS1 and ITS4 obtained about 550-700 bps DNA fragments. The acquired nucleotide sequences were deposited in the NCBI database and were used for BLAST search. The accession number and the BLAST analysis results were presented in Table 2. Phylogenetic analyses were carried out for the ITS sequence of each isolate and closely related species to confirm species determination (data not shown except for isolates from Polyporaceae; Fig. 1). The results corresponded to morphological identification of the samples. It was shown that 38 isolates were basidiomycetous macrofungi belonging to 22 species, 19 genera, 10 families and 4 orders. The families included Polyporaceae, Irpicaceae, Phanerochaetaceae, Agaricaceae, Psathyrellaceae, Physalacriaceae, Pleurotaceae, Strophariaceae, Stereaceae and Auriculariaceae. Most of the macrofungi (21 isolates) were identified as members of Polyporaceae including 10 and 8 isolates from Trametes and Ganoderma genera, respectively. Nine isolates were identified as ascomycetous microfungi and were considered as contamination of the macrofungal samples. It is found that two isolates GPS 002 and GPS 208 may be representatives of two novel taxa in Xylariaceae and Pleosporales, respectively.

In the phylogenetic tree constructed by ITS sequences of 21 isolates from Polyporaceae family (Fig. 1), the isolates were located in 4 distinct clades with high bootstrap values, corresponding the genera Ganoderma, Trametes, Fomes and Polyporellus.

In Ganoderma clade, 6 isolates located at the same position with an authentic strain of $G$. adspersum. The isolates can be divided into three groups based on ITS region sequence: (1) Bozchaft2, GPS 017 and GPS 047; (2) GPS 037 and GPS 038; and (3) GPS 052. ITS sequence of isolate GPS 052 differs from the sequences of group 1 and 2 by two and one nucleotide substitutions, respectively. Two other isolates in this clade were not identified at species level. The isolates were positioned at the same cluster with the authentic strains of G. lucidum, G. tsugae and G. oregonense. The ITS sequences of the isolates were different in four positions. 
In Trametes clade, it was shown that 10 isolates were conspecific with one of $T$. versicolor, $T$. gibbosa or T. hirsuta species. Among T. versicolor isolates, the sequence of GPS 107 differs from two other isolates by one nucleotide substitution. Two isolates of $T$. gibbosa showed one nucleotide variation. ITS sequences of three isolates of $T$. hirsuta were identical but different from the sequence of isolate GPS 119 by two nucleotides.

Among the remaining isolates of Polyporaceae family, two isolates were clustered with $F$. fomentarius and one other isolate was closely related to $P$. brumalis. ITS sequence of two isolates of $F$. fomentarius was different by one nucleotide substitution. Fomes fomentarius is a species complex containing four distinct clades. It seems that our isolate is more related to Chinese and South European clades but it was located at separate position from the clades.

Table 1 Sampling data and the results of initial morphological identification of the collected macrofungi.

\begin{tabular}{|c|c|c|c|c|}
\hline Forest & Location $^{*}$ & $\begin{array}{l}\text { Date of } \\
\text { sampling }\end{array}$ & $\begin{array}{l}\text { Macrofungi genera } \\
\text { (Morphologic identification) }\end{array}$ & $\begin{array}{l}\text { Selected isolates for } \\
\text { molecular } \\
\text { identification }\end{array}$ \\
\hline Nur & $\begin{array}{l}\text { N36 } 34 \text { E51 48, } \\
24-55 \text { m }\end{array}$ & 9/23/2015 & $\begin{array}{l}\text { Coprinellus, Ganoderma, } \\
\text { Lactarius, Mycena, Pleurotus, } \\
\text { Psathyrella, Trametes, } \\
\text { Volvariella, Xerula }\end{array}$ & $\begin{array}{l}\text { Nur 2, Nur 8, Nur 9, } \\
\text { Nur } 10\end{array}$ \\
\hline Royan & $\begin{array}{l}\text { N36 } 51 \text { E51 94, } \\
221-243 \text { m }\end{array}$ & 9/24/2015 & $\begin{array}{l}\text { Daedaleopsis, Irpex, Lactarius, } \\
\text { Lenzites, Leucoagaricus, } \\
\text { Omphalotus, Pleurotus }\end{array}$ & Royan 6 \\
\hline Bozchaft & $\begin{array}{l}\text { N36 } 38 \text { E52 76, } \\
186-192 \text { m }\end{array}$ & 4/18/2016 & Ganoderma, Trametes & Bozchaft 2 \\
\hline Darab Kola & $\begin{array}{l}\text { N36 } 29 \text { E53 18, } \\
661-860 \text { m }\end{array}$ & 4/20/2016 & $\begin{array}{l}\text { Collybia, Crepidotus, Cyclocybe, } \\
\text { Daldinia, Exidia, Fomes, } \\
\text { Ganoderma, Hypholoma, } \\
\text { Lenzites, Pleurotus, } \\
\text { Schizophyllum, Stereum, } \\
\text { Trametes }\end{array}$ & $\begin{array}{l}\text { Darabkola 1, } \\
\text { Darabkola 4, } \\
\text { Darabkola 8, } \\
\text { Darabkola 18, } \\
\text { Darabkola 21 }\end{array}$ \\
\hline Neka & $\begin{array}{l}\text { N36 } 33 \text { E53 23, } \\
190-941 \mathrm{~m}\end{array}$ & $4 / 21 / 2016$ & $\begin{array}{l}\text { Donkia, Ganoderma, Helvella, } \\
\text { Pleurotus, Trametes, Xylaria }\end{array}$ & Neka 24D, Neka 29-1 \\
\hline Nur & $\begin{array}{l}\text { N36 } 33 \text { E52 05, } \\
17-18 \mathrm{~m}\end{array}$ & $5 / 24 / 2016$ & $\begin{array}{l}\text { Coprinus, Crepidotus, } \\
\text { Ganoderma, Mycena, Trametes }\end{array}$ & $\begin{array}{l}\text { GPS 002, GPS 005, } \\
\text { GPS 016, GPS } 017\end{array}$ \\
\hline $\begin{array}{l}\text { Kashpel- } \\
\text { Lavij }\end{array}$ & $\begin{array}{l}\text { N36 } 23 \text { E52 02, } \\
\text { 331-692 m }\end{array}$ & $5 / 24 / 2016$ & Trametes & GPS 022 \\
\hline Si Sangan & $\begin{array}{l}\text { N36 } 34 \text { E51 48, } \\
55-58 \text { m }\end{array}$ & $5 / 25 / 2016$ & $\begin{array}{l}\text { Ceriporia, Crepidotus, Pleurotus, } \\
\text { Trametes }\end{array}$ & GPS 029 \\
\hline Dalkhani & $\begin{array}{l}\text { N36 } 82 \text { E50 66, } \\
\text { 650-801 m }\end{array}$ & $5 / 26 / 2016$ & $\begin{array}{l}\text { Ganoderma, Trametes, } \\
\text { Trichaptum }\end{array}$ & $\begin{array}{l}\text { GPS 037, GPS 038, } \\
\text { GPS 042, GPS 047, } \\
\text { GPS 052, GPS } 057\end{array}$ \\
\hline Chalus & $\begin{array}{l}\text { N36 } 62 \text { E51 42, } \\
257 \text { m }\end{array}$ & $5 / 27 / 2016$ & Trametes & GPS 063 \\
\hline $\begin{array}{l}\text { Abbas Abad- } \\
\text { Kelardasht }\end{array}$ & $\begin{array}{l}\text { N36 } 38 \text { E51 } 06 \\
375-416 \mathrm{~m}\end{array}$ & 10/26/2016 & $\begin{array}{l}\text { Ganoderma, Hypholoma, } \\
\text { Macrolepiota, Trametes }\end{array}$ & $\begin{array}{l}\text { GPS 101, GPS 106, } \\
\text { GPS } 107\end{array}$ \\
\hline Safa-Rud & $\begin{array}{l}\text { N36 } 39 \text { E53 35, } \\
382-567 \text { m }\end{array}$ & $10 / 27 / 2016$ & $\begin{array}{l}\text { Crepidotus, Fomes, Ganoderma, } \\
\text { Lycoperdon, Pholiota, Pleurotus, } \\
\text { Schizophyllum, Trametes, Xylaria }\end{array}$ & $\begin{array}{l}\text { GPS 119, GPS 122, } \\
\text { GPS 128, GPS 131, } \\
\text { GPS 142, GPS 146, } \\
\text { GPS } 158\end{array}$ \\
\hline Zirab-Lajim & $\begin{array}{l}\text { N36 14 E53 03, } \\
856-882 \mathrm{~m}\end{array}$ & $11 / 10 / 2016$ & $\begin{array}{l}\text { Armillaria, Bjerkandera, } \\
\text { Ganoderma, Hypholoma, } \\
\text { Polyporellus, Stropharia, } \\
\text { Trametes }\end{array}$ & $\begin{array}{l}\text { GPS 167, GPS 172, } \\
\text { GPS 173, GPS 177, } \\
\text { GPS 179, GPS } 180\end{array}$ \\
\hline $\begin{array}{l}\text { Abbas Abad- } \\
\text { Behshahr }\end{array}$ & $\begin{array}{l}\text { N36 } 39 \text { E53 35, } \\
382-511 \mathrm{~m}\end{array}$ & $11 / 11 / 2016$ & $\begin{array}{l}\text { Armillaria, Ganoderma, } \\
\text { Hericium, Hohenbuehelia, } \\
\text { Pleurotus, Xerula }\end{array}$ & $\begin{array}{l}\text { GPS 186, GPS 188, } \\
\text { GPS 196, GPS 197, } \\
\text { GPS } 208\end{array}$ \\
\hline
\end{tabular}


Table 2 The results of BLAST search for ITS region sequences of selected isolates and the related GenBank and IBRC-M accession numbers.

\begin{tabular}{|c|c|c|c|c|c|c|}
\hline Isolate & $\begin{array}{l}\text { IBRC- } \\
\text { M Acc. } \\
\text { No. }\end{array}$ & $\begin{array}{l}\text { Genbank } \\
\text { Acc. No. }\end{array}$ & Taxon & $\begin{array}{l}\text { Closest hit in } \\
\text { BLAST search }\end{array}$ & $\begin{array}{l}\text { Genbank } \\
\text { Acc. No. }\end{array}$ & Identity \\
\hline Bozchaft 2 & 30422 & MK050589 & Ganoderma sp. & G. adspersum & MG066632 & $100 \%$ \\
\hline \multirow[t]{2}{*}{ Darabkola 1} & \multirow[t]{2}{*}{30421} & \multirow[t]{2}{*}{ MK050606 } & \multirow[t]{2}{*}{ Stereum sp. } & S. armeniacum & MH862626 & \multirow{2}{*}{$100 \%$} \\
\hline & & & & S. hirsutum & KY628654 & \\
\hline Darabkola 4 & 30428 & MK050610 & Trametes hirsuta & T. hirsuta & JX501305 & $100 \%$ \\
\hline Darabkola 18 & 30354 & MK050586 & Exidia sp. ${ }^{*}$ & E. glandulosa & MF161201 & $99 \%$ \\
\hline Darabkola 21 & 30427 & MK050587 & Fomes fomentarius & F. fomentarius & LT629714 & $99 \%$ \\
\hline Nur 8 & 30409 & MK050607 & Trametes gibbosa & T. gibbosa & MH277950 & $100 \%$ \\
\hline Nur 9 & 30430 & MK050584 & Coprinellus sp. ${ }^{*}$ & C. disseminatus & JN159560 & $99 \%$ \\
\hline Nur 10 & 30431 & MK050605 & Psathyrella sp. ${ }^{*}$ & P. candolleana & MH856032 & $99 \%$ \\
\hline Neka 24D & 30310 & MK050585 & Donkia pulcherrima & D. pulcherrima & LC378994 & $99 \%$ \\
\hline \multirow[t]{2}{*}{ GPS 005} & \multirow[t]{2}{*}{30434} & \multirow[t]{2}{*}{ MK050600 } & \multirow[t]{2}{*}{ Irpex sp. ${ }^{*}$} & Irpex sp. & MH267976 & \multirow{2}{*}{$99 \%$} \\
\hline & & & & I. lacteus & MG554250 & \\
\hline GPS 017 & 30436 & MK050590 & Ganoderma sp. ${ }^{*}$ & G. adspersum & MG279153 & $100 \%$ \\
\hline GPS 022 & 30424 & MK050608 & Trametes gibbosa & T. gibbosa & MF161242 & $100 \%$ \\
\hline \multirow[t]{2}{*}{ GPS 029} & \multirow[t]{2}{*}{30437} & \multirow[t]{2}{*}{ MK050583 } & \multirow[t]{2}{*}{ Ceriporia sp. ${ }^{*}$} & Ceriporia sp. & KJ832049 & \multirow{2}{*}{$100 \%$} \\
\hline & & & & C. lacerata & KP135024 & \\
\hline GPS 037 & 30403 & MK050591 & Ganoderma sp. * & G. adspersum & JN588579 & $99 \%$ \\
\hline GPS 038 & 30405 & MK050592 & Ganoderma sp. $^{*}$ & G. adspersum & JN588579 & $99 \%$ \\
\hline GPS 042 & 30438 & MK050611 & Trametes hirsuta & T. hirsuta & JX501305 & $100 \%$ \\
\hline GPS 047 & 30439 & MK050593 & $\begin{array}{l}\text { Ganoderma } \\
\text { adspersum }\end{array}$ & G. adspersum & JN222417 & $100 \%$ \\
\hline GPS 052 & 30407 & MK050594 & Ganoderma sp. & G. adspersum & JN588579 & $99 \%$ \\
\hline GPS 057 & 30440 & MK050617 & Trichaptum sp. & T. biforme & FJ755247 & $99 \%$ \\
\hline GPS 063 & 30441 & MK050609 & Trametes gibbosa & T. gibbosa & KM373239 & $100 \%$ \\
\hline \multirow[t]{2}{*}{ GPS 101} & \multirow[t]{2}{*}{30327} & \multirow[t]{2}{*}{ MK050602 } & \multirow[t]{2}{*}{ Macrolepiota sp. } & M. konradii & JQ683125 & \multirow{2}{*}{$99 \%$} \\
\hline & & & & M. gracilenta & JQ683122 & \\
\hline GPS 106 & 30442 & MK050597 & $\begin{array}{l}\text { Hypholoma } \\
\text { fasciculare }\end{array}$ & H. fasciculare & JQ685719 & $99 \%$ \\
\hline GPS 107 & 30404 & MK050614 & Trametes sp. ${ }^{*}$ & T. versicolor & MF475935 & $100 \%$ \\
\hline GPS 119 & 30408 & MK050612 & Trametes sp. ${ }^{*}$ & T. hirsuta & KC589148 & $99 \%$ \\
\hline GPS 122 & 30443 & MK050615 & Trametes sp. ${ }^{*}$ & T. versicolor & МH320563 & $99 \%$ \\
\hline GPS 128 & 30444 & MK050613 & Trametes versicolor & T. versicolor & MF782818 & $100 \%$ \\
\hline
\end{tabular}


Table 2 Continued.

\begin{tabular}{|c|c|c|c|c|c|c|}
\hline Isolate & $\begin{array}{l}\text { IBRC- } \\
\text { M Acc. } \\
\text { No. }\end{array}$ & $\begin{array}{l}\text { Genbank } \\
\text { Acc. No. }\end{array}$ & Taxon & $\begin{array}{l}\text { Closest hit in } \\
\text { BLAST search }\end{array}$ & $\begin{array}{l}\text { Genbank } \\
\text { Acc. No. }\end{array}$ & Identity \\
\hline GPS 131 & 30313 & MK050588 & Fomes sp. $^{*}$ & F. fomentarius & KP641149 & $99 \%$ \\
\hline GPS 142 & 30316 & MK050603 & Pholiota sp. & P. aurivella & КТ355030 & $98 \%$ \\
\hline GPS 146 & 30411 & MK050599 & Irpex lacteus & I. lacteus & МH301114 & $100 \%$ \\
\hline GPS 158 & 30318 & MK050601 & Lycoperdon pyriforme & L. pyriforme & KP454030 & $100 \%$ \\
\hline GPS 167 & 30423 & MK050947 & Bjerkandera adusta & B. adusta & MH857085 & $100 \%$ \\
\hline GPS 172 & 30426 & MK050948 & Ganoderma sp. & G. lucidum & MG911000 & $99 \%$ \\
\hline GPS 173 & 30400 & MK050582 & Armillaria sp. ${ }^{*}$ & A. mellea & AF163583 & $99 \%$ \\
\hline GPS 177 & 30355 & MK050598 & Hypholoma sp. ${ }^{*}$ & H. fasciculare & KX449406 & $99 \%$ \\
\hline GPS 179 & 30399 & MK050616 & Trametes sp. * & T. hirsuta & MH910542 & $100 \%$ \\
\hline GPS 180 & 30324 & MK050604 & Polyporellus sp. ${ }^{*}$ & P. brumalis & KP283490 & $99 \%$ \\
\hline GPS 186 & 30334 & MK050595 & Ganoderma sp. & G. lucidum & MG911000 & $99 \%$ \\
\hline GPS 196 & 30342 & MK050596 & Hohenbuehelia sp. & $\begin{array}{l}\text { H. } \\
\text { auriscalpium } \\
\text { H. petaloides }\end{array}$ & $\begin{array}{l}\text { LN714552 } \\
\text { KT388021 }\end{array}$ & $99 \%$ \\
\hline
\end{tabular}

\begin{tabular}{|c|c|c|c|c|c|c|}
\hline \multicolumn{7}{|c|}{ Ascomycetous microfungi (contamination) } \\
\hline Darabkola 8 & 30429 & MK050620 & Nemania sp. ${ }^{*}$ & N. serpens & HM123484 & $100 \%$ \\
\hline Nur 2 & 30425 & MK050622 & & & & $100 \%$ \\
\hline Neka 29-1 & 30432 & MK050621 & Neopestalotiopsis sp. & $\begin{array}{l}\text { Neopestalotiop } \\
\text { sis sp. }\end{array}$ & MG649986 & $100 \%$ \\
\hline GPS 016 & 30435 & MK050623 & & & & $99 \%$ \\
\hline Royan 6 & 30433 & MK050619 & Didymosphaeria sp. & & & \\
\hline GPS 188 & 30311 & MK050618 & $\begin{array}{l}\text { (Paraconiothyrium } \\
\text { brasiliense) }\end{array}$ & P. brasiliense & MH532510 & $99 \%$ \\
\hline GPS 197 & 30416 & MK050625 & Pochonia sp. $^{*}$ & $\begin{array}{l}P . \\
\text { chlamydospori } \\
a\end{array}$ & AB709845 & $100 \%$ \\
\hline GPS 002 & 30446 & MK050626 & Xylariaceae sp. & Xylariaceae sp. & JQ761922 & $98 \%$ \\
\hline GPS 208 & 30445 & MK050624 & Pleosporales sp. & $\begin{array}{l}\text { Uncultured } \\
\text { Pezizomycotina }\end{array}$ & KT581743 & $98 \%$ \\
\hline
\end{tabular}

\footnotetext{
* Species delineation using phylogenetic analyses for the strains were matched to the closest hit in BLAST search. However, the results cautiously were not submitted.
} 


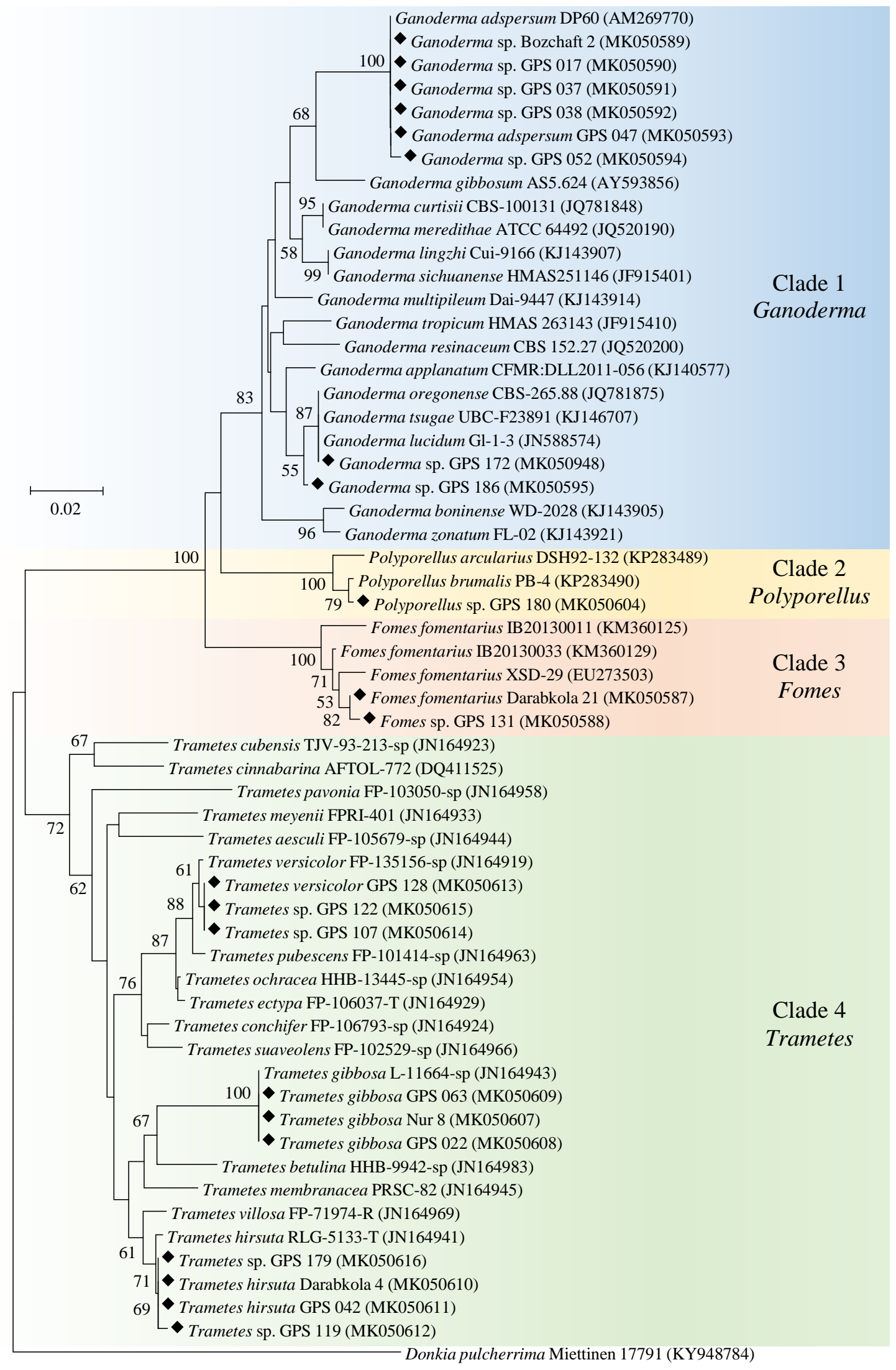

Fig. 1 - Phylogenetic tree based on ITS region (including 5.8S rRNA gene) sequence showing the position of macrofungal strains in Polyporaceae family among the related species. The phylogram was constructed from evolutionary distance data with Kimura's two-parameter correction, using the neighbor-joining method. Donkia pulcherrima Miettinen 17791 was the designated outgroup for the analysis. Bootstrap values above 50\% (based on 1000 replicates) are given at branch points. Bar, 0.02 substitutions per nucleotide position. 


\section{Discussion}

Study on diversity of wild populations of edible and medicinal macrofungi provides opportunities to apply their beneficial properties. There are several reports on collection and morphological characterization of wild macrofungi from Iran, especially Northern Iran (Amoopour et al. 2016, Asef 2008, Asef \& Muradov 2012, Borhani et al. 2010, Karim et al. 2012, 2013, Moradali et al. 2007, Olfati et al. 2009). In this study, 38 macrofungi genera were identified based on initial morphological features from Mazandaran province. In a checklist of Iranian non-gilled/non-gasteroid hymenomycetes published by Ghobad-Nejhad \& Hallenberg (2012), Mazandaran province had the second highest diversity in Iran including 214 species from 128 genera. Borhani et al. (2010) reported 100 species from 57 genera from this province. Most of collected genera of this study have been reported previously from Mazandaran province; among them two genera Hohenbuehelia and Polyporellus can be considered as new records for Iran. The identity of the samples was confirmed by ITS sequencing and phylogenetic analysis. The isolate GPS 196 was collected from Abbas Abad forest and showed highest ITS sequence similarity (99\%) to $H$. auriscalpium and $H$. petaloides. Further study using multi-locus sequence analysis is needed for identification of the isolate at species level. The isolate GPS 180 was collected from a forest in Zirab-Lajim region. The ITS sequence of the isolated was $99 \%$ similar to the related sequence from $P$. brumalis strains and located at the same clade with them in the phylogenetic tree (Fig. 1). Polyporellus brumalis have been mentioned in excluded taxa of the checklist presented by Ghobad-Nejhad \& Hallenberg (2012). In addition, isolate GPS 029 was closely related to Ceriporia lacerata based on NCBI and CBS BLAST searches and phylogenetic analysis of the ITS sequence. The species have not been reported previously from Iran in the available literatures.

Morphological and ecological characterization have been used by most of studies on biodiversity of macrofungi in Iran (Amoopour et al. 2016, Asef 2008, Asef \& Mudarov 2012, Borhani et al. 2010, Ghobad-Nejhad \& Langer 2016, Karim et al. 2012, 2013, Olfati et al. 2009). There are few reports for molecular identification of wild populations of Iranian macrofungi using ITS region sequence analysis (Ghobad-Nejhad \& Langer 2016, Rezaeian et al. 2015, Tajalli et al. 2015). Therefore, there is a lack of molecular data for Iranian macrofungi in available databases. In this study, 38 ITS region sequences from 22 species were obtained and deposited on GenBank. ITS sequencing and phylogenetic analysis were successfully applied for taxon determination. However, further studies using other gene markers is required for species identification at species level in some cases (Table 2). For example, translation elongation factor 1- $\alpha$ (tef1- $\alpha$ ) and the second largest subunit of RNA polymerase II (rpb2) loci have been applied for classification of Ganoderma species (Matheny et al. 2007, Jargalmaa et al. 2017). It was demonstrated that the combination of morphological and molecular (ideally multi-locus) analysis can resolve the problems related to taxonomy of fungi (Jung et al. 2014, Jargalmaa et al. 2017).

Macrofungi have great importance in food, medicine and cosmetics. Several beneficial properties have been reported for most of species studied in this study (Agrawal et al. 2017, Ahmad 2018, Cespedes et al. 2015, Doskocila et al. 2016, Dresch et al. 2015, Dyakov et al. 2011, El Enshasy \& Hatti-Kaul 2013, Reis et al. 2017, Tang et al. 2018, Tel-Çayan et al. 2015, Yang et al. 2013, Yin et al. 2014). Some isolates were closely related to medicinally important macrofungi including Ganoderma lucidum, G. adspersum, Trametes versicolor, T. hirsuta, T. gibbosa, Fomes fomentarius, Armillaria mellea, Irpex lacteus and Stereum hirsutum. Pure mycelial cultures of the macrofungi are available in IBRC-M culture collection and can be used to evaluate their potential applications. Collection and morphological identification of some Ganoderma species from Iran have been reported by Moradali et al. (2007) and Keypour et al. (2014). They reported that Iranian Ganoderma species include G. applanatum, G. adspersum, G. colossus, G. lucidum, G. resinaceum, G. tsugae and G. manoutchehrii. Heydarian \& Hatamian-Zarmi (2016) identified an Iranian G. lucidum strain (HA2012-001) using ITS sequencing for the first time. The strain was isolated from Dohezar forest in Mazandaran province. The ITS sequence of the strain (GenBank accession number KX765192) differs from our strains GPS 172 and GPS 186 by two and four nucleotide substitutions, respectively. Therefore, they are different strains. ITS sequence variation was also observed among our strains 
belonging to G. adspersum, T. versicolor, T. hirsuta T. gibbosa and I. lacteus strains isolated from different forests in Mazandaran province. These data indicate great diversity of macrofungi in Northern Iran. Beside biodiversity evaluations, precise identification by molecular methods is important from applied point of view due to species-specific and strain-specific production of bioactive compounds by medicinal macrofungi.

\section{Acknowledgements}

This study was conducted as part of a research project financed by ACECR, Iran (code no 2283) granted to Dr. HR Pourianfar and Dr. MA Amoozegar.

\section{References}

Agrawal DC, Tsay HS, Shyur LF, Wu YC, Wang SY. 2017 - Medicinal Plants and Fungi: Recent Advances in Research and Development. Springer, Singapore.

Ahmad MF. 2018 - Ganoderma lucidum: Persuasive biologically active constituents and their health endorsement. Biomedicine and Pharmacotherapy 107, 507-519.

Amoopour M, Ghobad-Nejhad M, Khodaparast SA. 2016 - New records of polypores from Iran, with a checklist of polypores for Gilan Province. Czech Mycology 68, 139-148.

Asef MR. 2008 - Macrofungi flora of Arasbaran. 2. Bolete fungi (Families Boletaceae and Suillaceae). Rostaniha 9, 210-229.

Asef MR, Etemad V. 2016 - Identification of agaric fungi of Kheyroud Research Forest, Noshahr (Mazandaran province, N. Iran). Rostaniha 17, 19-27.

Asef MR, Muradov P. 2012 - Lepiotaceous fungi (Agaricaceae) in the Iranian part of Caucasia. Turkish Journal of Botany 36, 289-294.

Bisen PS, Debnath M, Prasad GBKS. 2012 - Microbes: Concepts and Applications. Wiley Online Library, Hoboken, pp. 275-338.

Borhani A, Badalyan SM, Garibyan NN, Mosazadeh SA. 2010 - Diversity and distribution of macrofungi associated with beech forests of northern Iran (case study Mazandaran Province). World Applied Sciences Journal 11, 151-158.

Cespedes CL, Alarcon J, Aqueveque PM, Lobo T et al. 2015 - New environmentally-friendly antimicrobials and biocides from Andean and Mexican biodiversity. Environmental Research 142, 549-562.

Cheung PCK. 2010 - The nutritional and health benefits of mushrooms. British Nutrition Foundation Nutrition Bulletin 35, 292-299.

Dentinger BT, Didukh MY, Moncalvo JM. 2011 - Comparing COI and ITS as DNA barcode markers for mushrooms and allies (Agaricomycotina). PLoS One 6, e25081.

Doskocil I, Havlik J, Verlotta R, Tauchen J et al. 2016 - In vitro immunomodulatory activity, cytotoxicity and chemistry of some central European polypores. Pharmaceutical Biology 54, 2369-2376.

Dresch P, D’Aguanno MN, Rosam K, Grienke U et al. 2015 - Fungal strain matters: colony growth and bioactivity of the European medicinal polypores Fomes fomentarius, Fomitopsis pinicola and Piptoporus betulinus. AMB Express 5, 4.

El Enshasy HA, Hatti-Kaul R. 2013 - Mushroom immunomodulators: unique molecules with unlimited applications. Trends in Biotechnology 31, 668-677.

Felsenstein J. 1985 - Confidence limits on phylogenies: an approach using the bootstrap. Evolution 39, 783-791.

Ghobad-Nejhad M, Langer E. 2016 - First inventory of aphyllophoroid basidiomycetes of Zagros forests, W Iran. Plant Biosystems 151, 844-854.

Ghobad-Nejhad M, Hallenberg N. 2012 - Checklist of Iranian non-gilled/non-gasteroid hymenomycetes (Agaricomycotina). Mycotaxon 119: 493-494. 
Hawksworth DL. 2012 - Global species number of fungi: Are tropical studies and molecular approaches contributing to a more robust estimate? Biodiversity and Conservation 21, 24252433.

Heydarian M, Hatamian-Zarmi A. 2016 - Molecular identification of Ganoderma lucidum from Iran. Rostaniha 17, 188-192.

Jargalmaa S, Eimes JA, Park MS, Park JY et al. 2017 - Taxonomic evaluation of selected Ganoderma species and database sequence validation. PeerJ 5, e3596.

Jung PE, Fong JJ, Park MS, Oh SY et al. 2014 - Sequence validation for the identification of the white-rot fungi Bjerkandera in public sequence databases. Journal of Microbiology and Biotechnology 24, 1301-1307.

Karim M, Kavosi MR, Hajizadeh G. 2013 - Macro-fungal communities in Hyrcanian forests, North of Iran: relationships with season and forest types. Ecologia Balkania 5, 87-96.

Karim M, Kavosi MR, Mosazadeh SA, Borhani A. 2012 - Study on diversity and frequency of macrofungi in deciduous and mix forestation of Northern Iran (Case study, Golestan province). World Applied Sciences Journal 19, 1268-1272.

Keypour S, Riahi H, Safaie N, Borhani A. 2014 - Mycelial growth rate and macro- and micromorphological characteristics of medicinal species of genus Ganoderma (Higher Basidiomycetes) from Iran. International Journal of Medicinal Mushrooms 16, 365-374.

Kumar S, Stecher G, Tamura K. 2016 - MEGA7: Molecular evolutionary genetics analysis version 7.0 for bigger datasets. Molecular Biology and Evolution 33, 1870-1874.

Dyakov MY, Kamzolkina OV, Shtaer OV, Bis'ko NA et al. 2011 - Morphological characteristics of natural strains of certain species of Basidiomycetes and biological analysis of antimicrobial activity under submerged cultural conditions. Microbiology 80, 274-285.

Masoumi F, Pourianfar HR, Masoumi A, Mostafavi Mendi E. 2015 - A study of mycelium characterization of several wild genotypes of the button mushroom from Iran. International Journal of Advanced Research 3, 236-246.

Matheny PB, Wang Z, Binder M, Curtis JM et al. 2007 - Contributions of rpb2 and tef1 to the phylogeny of mushrooms and allies (Basidiomycota, Fungi). Molecular Phylogenetics and Evolution 43, 430-451.

Money NP. 2016 - Are mushrooms medicinal? Fungal Biology 120, 449-453.

Moradali MF, Hedjaroude GA, Mostafavi H, Abbasi M et al. 2007 - The genus Ganoderma (Basidiomycota) in Iran. Mycotaxon 99, 251-269.

Moser M. 1983 - Keys to Agarics and Boleti (Polyporales, Boletales, Agaricales, Russulales). Roger Phillips, London.

Olfati J, Peyvast G, Mami Y. 2009 - Identification and chemical properties of popular wild edible mushrooms from northern Iran. Journal of Horticulture and Forestry 1, 48-51.

Rathore H, Prasad S, Sharma S. 2017 - Mushroom nutraceuticals for improved nutrition and better human health: A review. Pharma Nutrition 5, 35-46.

Redhead S. 1997 - Macrofungi of British Columbia: requirements for inventory. Research Branch, B.C. Ministry of Forests, and Wildllife Branch, B.C. Ministry of Environment, Lands, and Parks, Victoria, B.C. Work. Pap. 28/1997.

Reis FS, Martins A, Vasconcelos MH, Morales P, Ferreira ICFR. 2017 - Functional foods based on extracts or compounds derived from mushrooms. Trends in Food Science and Technology 66, $48 \mathrm{e} 62$.

Rezaeian S, Pourianfar HR, Janpoor J. 2016 - Collection and identification of Iranian wild mushrooms: towards establishment of a mushroom bio-bank. International Journal of Advanced Research 4, 256-260.

Rezaeian S-H, Saadatmand S, NejadSattari T, Mirshamsi A. 2015 - Antioxidant potency of Iranian newly cultivated wild mushrooms of Agaricus and Pleurotus species. Biomedical Research 26, 534-542.

Roncero-Ramos I, Delgado-Andrade C. 2017 - The beneficial role of edible mushrooms in human health. Current Opinion in Food Science 14, 122-128. 
Roupas P, Keogh J, Noakes M, Margetts C, Taylor P. 2012 - The role of edible mushrooms in health: Evaluation of the evidence. Journal of Functional Foods 4, 687-709.

Ryvarden L. 1991 - Genera of polypores, nomenclature and taxonomy. Synopsis Fungorum 5, 1373.

Ryvarden L, Melo I. 2014 - Poroid fungi of Europe. Synopsis Fungorum. 31, 1-455.

Saba F, Papizadeh M, Khansha J, Sedghi M et al. 2016 - A rapid and reproducible genomic DNA extraction protocol for sequence-based identification of archaea, bacteria, cyanobacteria, diatoms, fungi, and green algae. Journal of Medical Bacteriology 5, 22-28.

Schoch CL, Seifert KA, Huhndorf S, Robert V et al. 2012 - Nuclear ribosomal internal transcribed spacer (ITS) region as a universal DNA barcode marker for Fungi. Proceedings of the National Academy of Science (PNAS) 109, 6241-6246.

Sivanandhan S, Khusro A, Paulraj MP, Ignacimuthu S, AL-Dhabi NA. 2017 - Biocontrol properties of Basidiomycetes: An overview. Journal of Fungi 3, 2.

Tajalli F, Malekzadeh KH, Soltanian H, Janpoor J et al. 2015 - Antioxidant capacity of several Iranian, wild and cultivated strains of the button mushroom. Brazilian Journal of Microbiology 46, 769-776.

Tang Y, Zhao ZZ, Li ZH, Feng T et al. 2018 - Irpexoates A-D, four triterpenoids with malonyl modifications from the fruiting bodies of the medicinal fungus Irpex lacteus. Natural Products and Bioprospecting 8, 171-176.

Taofiq O, González-Paramás AM, Martins A, Barreiro MF, Ferreira ICFR. 2016 - Mushrooms extracts and compounds in cosmetics, cosmeceuticals and nutricosmetics - A review. Industrial Crops and Products 90, 38-48.

Tel-Çayan G, Öztürk M, Duru ME, Rehman MU et al. 2015 - Phytochemical investigation, antioxidant and anticholinesterase activities of Ganoderma adspersum. Industrial Crops and Products 76, 749-754.

Thompson JD, Higgins DG, Gibson TJ. 1994 - CLUSTAL W: improving the sensitivity of progressive multiple sequence alignment through sequence weighting, position-specific gap penalties and weight matrix choice. Nucleic Acids Research 22, 4673-4680.

Wasser SP. 2014 - Medicinal mushroom science: Current perspectives, advances, evidences, and challenges. Biomedical Journal 37, 345-356.

Wasser SP. 2010 - Medicinal mushroom science: History, current status, future trends, and unsolved problems. International Journal of Medicinal Mushrooms 12, 1-16.

White TJ, Bruns TD, Lee SB, Taylor JW. 1990 - Amplification and direct sequencing of fungal ribosomal RNA genes for phylogenetics. In: Innis MA, Gelfand DH, Sninsky JJ, White TJ, (eds), PCR Protocols: a guide to methods and applications. Academic Press, New York, pp. 315-322.

Yang XY, Feng T, Ding JH, Li ZH et al. 2013 - Two new drimane sesquiterpenoids from cultures of the basidiomycete Trichaptum biforme. Natural Products and Bioprospecting 3, 154-157.

Yin X, Feng T, Li ZH, Leng Y, Liu JK. 2014 - Five new guanacastane-type diterpenes from cultures of the fungus Psathyrella candolleana. Natural Products and Bioprospecting 4, 149-155. 